\title{
Enfoque de Género en la atención de salud de pacientes con enfermedades cardiovasculares en Chile
}

\author{
Lizet Véliz ${ }^{1}$, Andrés Bianchetti ${ }^{2}$. \\ 1. Departamento de Enfermería. Universidad de La Serena, Chile. \\ 2. Facultad de Ciencias de la Salud. Universidad San Sebastián, Chile.
}

\section{Sr. Editor:}

El enfoque de género en el tratamiento y cuidados de pacientes con enfermedades cardiovasculares ha sido una condición que no se ha formalizado de un modo explícito en las guías clínicas que orientan las atenciones de los pacientes que presentan estas patologías en Chile ${ }^{1}$. En los últimos años, el número de pacientes afectados por enfermedades crónicas no transmisibles (ECNT) ha ido en aumento $^{1}$, lo que ha transformado a este grupo objetivo en un desafío para las intervenciones que desde la salud pública se diseñan para controlar sus patologías. En este contexto, es necesario incorporar el enfoque de género en la atención de salud de los pacientes con ECNT de un modo explícito, de manera de aumentar la efectividad de los cuidados al realizar un tratamiento que goce de mayor pertinencia cultural, tanto en la relación terapéutica como en el ajuste a su realidad sociocultural. La protocolización de la incorporación del enfoque de género en la atención de pacientes con enfermedades cardiovascu- lares permitiría masificar su aplicación por parte de los profesionales del ámbito sanitario sin que su uso quede determinado sólo por las competencias y criterio del profesional, como sucede actualmente. Adicionalmente, esta incorporación normada del enfoque de género estaría contribuyendo transversalmente a la construcción de una sociedad más justa y equitativa en términos del acceso y respuesta desde las instituciones de salud para los hombres y las mujeres. Vinculado a lo anterior, la OPS señala que el enfoque de género es esencial para abordar la problemática en salud, atendiendo a las necesidades particulares de mujeres y hombres, y reconociendo no sólo sus diferencias biológicas, sino también especificidades propias de la construcción social del género ${ }^{2}$.

En diferentes regiones del mundo se ha encontrado que los factores de riesgo cardiovasculares (RCV) son, en general, similares para hombres y mujeres. Sin embargo, a través del tiempo se han demostrado diferencias de género en la mayoría de ellos, en cuanto a su prevalencia, 
relación con otros factores de riesgo y la presencia de los determinantes sociales de la salud ${ }^{3}$.

Las diferencias de género en la enfermedad cardiovascular incluyen aspectos patofisiológicos, manifestaciones clínicas, percepción de la enfermedad e intervención y tratamiento de éstas, lo cual determina una evolución y desenlaces diferentes ${ }^{3}$. Considerando estas diferencias, uno de los objetivos de la actual política en salud es alcanzar la equidad en salud en general, como también la equidad de género de manera transversal a lo largo del curso de la vida ${ }^{4}$.

Especificando las diferencias aludidas, para el sexo masculino la construcción social de la masculinidad podría condicionar en forma negativa su calidad de vida, sus redes sociales, las modalidades de enfermar y de cuidar su salud ${ }^{4}$. Los indicadores sanitarios disponibles en Chile muestran que los hombres se mueren antes y se enferman más que las mujeres ${ }^{4}$. También existe una mayor tasa de inasistencia a los controles de salud cardiovascular en atención primaria por parte de los hombres, especialmente los más jóvenes que se encuentran en edad productiva (Consulta a expertos: Departamento de enfermedades crónicas no transmisibles. MINSAL. 05 de Noviembre 2012).

En el caso de las mujeres, autores señalan que aquellas con enfermedades cardiovasculares presentan, en la confrontación de las condiciones de vida, el "síndrome de la supermujer", es decir, que se perciben y actúan como imprescindibles. Con respecto a las propias expectativas, esto las expone a situaciones sin salida, que generan mucha angustia en subjetividades que manifiestan mucha dificultad para su manejo. En esta misma línea, se ha indicado que no es sólo la entrada a la vida de mujer moderna y al mercado laboral asalariado en condiciones "masculinas" lo que ha constituido un estresor específico para las mujeres, sino la sumatoria de áreas de las cuales ocuparse: trabajo, familia, desarrollo personal, requerimientos estéticos, entre otros. Se establece así un perfil específico del síndrome de la supermujer que, en lugar de flexibilizar ideales y articular áreas, establece una sumatoria de aspiraciones que generan una sobrecarga emocional y física 5 .

Las barreras de mayor impacto para las mujeres, detectadas hasta el momento, corresponden a minorías raciales y étnicas, menor nivel escolar, baja autoestima, menor nivel socioeconómico, mayor edad, exceso de responsabilidades, menor soporte familiar y social, desconocimiento del riesgo personal, desconocimiento de los factores de riesgo, confusión en la información recibida y subvaloración del riesgo de enfermedad cardiovascular por parte del personal de salud ${ }^{5}$.

Considerando lo anterior, es una responsabilidad de la salud pública abordar sus necesidades y contribuir a eliminar las barreras que existen entre hombres y mujeres para disminuir las desventajas en la implementación de estilos de vida saludables, tales como mejorar la actividad física, reducir el inicio del consumo de tabaco, favorecer el cese de este, elegir alimentación más saludable y manejar el estrés, elementos claves en el tratamiento de las enfermedades cardiovasculares. En esta línea, protocolizar la aplicación del enfoque de género en las atenciones de salud de los pacientes con enfermedades cardiovasculares resulta fundamental para colaborar en la obtención de un resultado exitoso a nivel terapéutico, tanto desde la promoción como en el tratamiento de las enfermedades cardiovasculares en Chile. 


\section{Referencias:}

1.- Ministerio de salud de Chile (MINSAL).Departamento enfermedades no transmisibles. Implementación del enfoque de riesgo en el programa de salud cardiovascular [Internet]. 2010 [Citado 04 de octubre 2012]. Disponible en: http:// www.minsal.gob.cl/portal/url/item/787e4765248bc9e0e04001011f0172b5.pdf

2.- Organización Panamericana de la Salud (OPS). Comprender la salud de los hombres. Un enfoque relacional y sensible al género [Internet]. 2000 [Citado 19 de Enero del 2016]. Disponible en: http://iris.paho.org/xmlui/bitstream/handle/123456789/804/9275322848.pdf?sequence $=1$

3.- Vaccarino V, Badimon L, Corti R, de Wit C, Dorobantu M, Hall
A, et al. Ischaemic heart disease in women: are there sex differences in pathophysiology and risk factors? Cardiovascular Research 2011; 90 9-17.

4.- Ministerio de salud de Chile (MINSAL). Situación actual de la salud del hombre en Chile [Internet]. 2011 [Citado 19 de Enero del 2016]. Disponible en: http://web.minsal.cl/portal/url/item/ b7e8f68be82d7f2fe040010165013351.pdf

5.- Tajer D. Mujeres y enfermedad cardiovascular. Género y subjetividad en la construcción del riesgo en enfermedades cardiovasculares en mujeres jóvenes. Anuario de investigaciones UBA. 2005; 13: 239-248. 